\title{
Le contrôle migratoire aux frontières Schengen : pratiques et représentations des polices sur la ligne tchéco-autrichienne
}

Migration control at Schengen borders: Police's practices and representations on the Czech-Austrian borderline

El control de las migraciones en las fronteras Schengen: prácticas y

representaciones de la policía en la frontera entre la República Checa y Austria

\section{Mathilde Darley}

\section{(2) OpenEdition}

\section{Journals}

\section{Édition électronique}

URL : http://journals.openedition.org/conflits/16583

DOI : $10.4000 /$ conflits. 16583

ISSN : $1777-5345$

Éditeur :

CCLS - Centre d'études sur les conflits lilberté et sécurité, L'Harmattan

Édition imprimée

Date de publication : 30 octobre 2008

Pagination : 13-29

ISBN : 978-2-296-06624-3

ISSN : 1157-996X

Référence électronique

Mathilde Darley, «Le contrôle migratoire aux frontières Schengen : pratiques et représentations des polices sur la ligne tchéco-autrichienne », Cultures \& Conflits [En ligne], 71 | automne 2008, mis en ligne le 05 février 2009, consulté le 30 mars 2021. URL : http://journals.openedition.org/conflits/16583 ; DOI : https://doi.org/10.4000/conflits. 16583 


\title{
Le contrôle migratoire aux frontières Schengen : pratiques et représentations des polices sur la ligne tchéco-autrichienne
}

\author{
Mathilde DARLEY
}

Mathilde Darley est post-doctorante Lavoisier au Centre Marc Bloch à Berlin. Elle est titulaire d'une thèse de doctorat à l'IEP de Paris (Frontière, asile et détention des étrangers. Le contrôle étatique de l'immigration et son contournement en Autriche et en République tchèque). Ses recherches portent sur le contrôle migratoire, l'enfermement des étrangers, la traite et la prostitution. Elle a notamment publié "La prostitution en clubs dans les régions frontalières de la République tchèque », Revue française de sociologie, vol. 48, $n^{\circ} 2$, avril-juin 2007, pp. 273-306.

T es postes frontières sont sans doute la manifestation la plus évidente de Ll'implantation dans l'espace d'un élément de discontinuité (le poste et son aménagement architectural) explicitement affecté au contrôle des individus souhaitant entrer sur le territoire national ou en sortir. Souvent premiers points de contact du voyageur avec l'Etat sur le territoire duquel il pénètre et, à ce titre, premiers représentants de l'Etat-nation, les gardes-frontières incarnent l'ordre étatique légitime ${ }^{1}$. A travers la matérialisation architecturale du poste frontière, l'Etat donne à voir le contrôle qu'il entend exercer sur son territoire par le filtrage des voyageurs cherchant à y pénétrer (ou à en sortir). De ce fait, les frontières ont souvent été présentées, notamment dans les nombreux travaux de recherche développés sur ce thème depuis le début des années 1990, comme le lieu privilégié d'investigation et d'interprétation des aspects symboliques de l'Etat, puisque s'y exerce "l'autorité souveraine de l'Etat d'exclure 2 ». A cela s'ajoute le rôle croissant qu'on leur reconnaît géné-

1. Schwell A., "Unequal Partners. Security and Identity at the Polish-German Border", Research Group Transnationalism Working Paper, $\mathrm{n}^{\circ} 8,2006$, p. 3. Disponible sur : http://publikationen.ub.uni-frankfurt.de/volltexte/2007/3672/

2. Voir Geddes A., “Europe’s Border Relationships and International Migration Relations”, Journal of Common Market Studies, vol. 43, n4, 2005, pp. 787-806 (p. 788). Notre traduction. 
ralement dans le dispositif politique, dans un contexte d'évolution supposée des risques sécuritaires vers des formes de plus en plus transfrontalières et non plus «stato-centrées 3 ».

Malgré la dimension hautement symbolique des postes frontières comme remparts de la souveraineté nationale, ceux-ci n'ont que très rarement fait l'objet d'études empiriques, sans doute en raison du caractère fermé qu'on leur prête. Lieux de passage, mais aussi points de contact et/ou de friction entre Etats et étrangers, leur observation renseigne pourtant sur la manière dont le contrôle et les modes de son contournement interagissent au niveau local, voire national. Alors que les nombreux travaux de recherche disponibles sur le contrôle migratoire s'articulent souvent autour de l'hypothèse d'un fossé grandissant entre les politiques migratoires et leur mise en ouvre (gap theory) ${ }^{4}$, nous avons cherché à complexifier cette analyse en dépassant le niveau d'observation macrosociale qui la sous-tend généralement : au-delà de l'étude des politiques publiques étatiques, qui conduit souvent à négliger la diversité des pratiques locales découlant ou débordant du cadre juridico-politique établi, il s'agit ici de chercher à saisir le pouvoir de l'Etat tel qu'il est mis en ouvre. Pour ce faire, nous nous appuyons sur les observations ethnographiques conduites entre 2004 et 2007 au sein de postes frontières situés à la frontière tchéco-autrichienne ${ }^{5}$. En effet, l'Autriche et la République tchèque, séparées jusqu'en 2004 par une frontière externe de l'Union européenne et, entre 2004 et 2007, par une frontière externe de l'espace Schengen (levée fin 2007), semblent fournir un terrain privilégié d'observation du passage entre l'Est et l'Ouest de l'Europe, mais également des transformations de la frontière Schengen, espace de cristallisation des efforts de contrôle de l'immigration irrégulière en Europe.

\section{Symbolique de la frontière dans les politiques migratoires tchèques et autrichiennes}

Dès 1990, alors même que la plupart des Etats post-communistes voisins, fraîchement indépendants, se donnent pour mission de transférer à l'administration civile le contrôle de leurs frontières nationales, jusqu'alors assuré par

3. Lutterbeck D., "The New Security Agenda and the Rise of Gendarmeries", Cooperation and Conflict, vol. 39, ${ }^{\circ} 1,2004$, pp. 45-68 (p. 51). Notre traduction.

4. Voir par exemple Cornelius W.A., Tsuda T., Martin P.L., Hollifield J.F. (eds.), Controlling

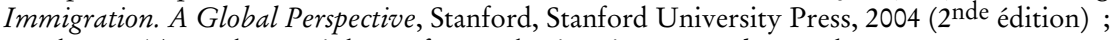
Castles S., "Towards a Sociology of Forced Migration", Sociology, vol. 37, n¹, 2003, pp. 1334 ; Sassen S., Losing Control? Sovereignty in an Age of Globalization, New York, Columbia University Press, 1996 ; Bigo D., Guild E. (eds.), Controlling Frontiers. Free Movement into and within Europe, Hants / Burlington, Ashgate Publishing Limited, 2005.

5 . Ces observations ont été conduites dans le cadre d'une thèse de doctorat portant sur le contrôle migratoire en Autriche et en République tchèque. Voir Darley M.A., Frontière, asile et détention des étrangers. Le contrôle étatique de l'immigration et son contournement en Autriche et en République tchèque, thèse de doctorat à l'Institut d'études politiques sous la direction de G. Mink, Paris, 2008. 
l'armée, le Conseil des ministres autrichiens décide d'impliquer l'armée dans la surveillance des frontières nationales ${ }^{6}$. Cette décision, initiée par le ministère de l'Intérieur et motivée par la nécessité invoquée d'accroître très rapidement l'ampleur et l'efficacité du contrôle frontalier pour faire face à l'augmentation des flux migratoires en provenance des pays d'Europe de l'Est, est dans un premier temps limitée dans l'espace (elle ne concerne qu'une portion de la frontière austro-hongroise) et dans le temps (la participation de l'armée au contrôle frontalier est initialement prévue pour dix semaines seulement). Toutefois, alors que les médias et certains partis politiques agitent le spectre d'une arrivée - décrite en termes invasionnels - de migrants clandestins de l'Est sur le territoire autrichien, la décision est reconduite et le territoire d'intervention de l'armée étendu. Ainsi, depuis 1990, cette décision à l'origine exceptionnelle du Conseil des ministres a été prolongée d'un an chaque année, et l'armée participe désormais au contrôle de 455 kilomètres de frontières avec la Slovaquie et la Hongrie. Si les militaires affectés au contrôle des frontières se trouvent alors sous la responsabilité du ministère de l'Intérieur et non plus du ministère de la Défense, cette coopération entre police et armée pour le contrôle aux frontières fait malgré tout de l'Autriche un cas unique à l'échelle européenne. Bien que nos enquêtes ne nous aient pas permis d'observer les formes locales d'association de l'armée au contrôle des frontières, la frontière tchéco-autrichienne n'étant pas concernée par cette mesure, le choix de placer du personnel en uniforme militaire (traditionnellement affecté à la défense du pays contre une éventuelle agression extérieure) sur une partie des frontières autrichiennes peut être interprété comme une démonstration symbolique, par l'Etat, du contrôle qu'il prétend détenir sur son territoire.

Au-delà du symbole, la présence de l'armée aux frontières est quantitativement importante ${ }^{7}$ et jouit d'une visibilité médiatique forte : ainsi, en 1999, l'armée autrichienne organise une importante campagne de publicité dans la presse nationale pour réclamer une augmentation de son budget. Les annonces, qui occupent une demi-page des plus grands quotidiens autrichiens, comportent une photo, représentant un jeune homme, les mains en l'air, fouillé par un soldat, surmontée de l'inscription, en caractères rouges et gras, « on appelle à l'aide quand les illégaux franchissent nos frontières » et accompagnée du texte suivant :

« Notre armée est là et montre ce dont elle est capable. Y compris quand il en va de la protection de notre démocratie, de notre liberté et de notre sécurité. Depuis neuf ans, nos soldats sont postés à la frontière nationale dans la région du Burgenland. 2000 hommes

6. Décision du Conseil des ministres du 4 septembre 1990.

7. Environ 2200 militaires et 3000 fonctionnaires de la police des frontières sont actuellement affectés au contrôle frontalier. De 1990 à 2004, environ 290000 soldats ont participé au contrôle des frontières nationales. 
nuit et jour. Par tous les temps. Qui arrêtent les trois-quarts des migrants illégaux. Notre armée a protégé. Et s’il le faut, nos soldats protégeront encore ${ }^{8}$. »

Quoique géographiquement périphérique, la frontière est donc ici au cœur du politique, premier garant de la protection de la démocratie, de la liberté et de la sécurité de la société autrichienne. C’est pourquoi la police et l'armée lancent régulièrement des appels à la population des régions frontalières, incitée par le biais de tracts à participer à la protection des frontières et à la lutte contre l'immigration illégale 9 . Les gardes-frontières autrichiens rencontrés lors des enquêtes de terrain insistent d'ailleurs sur le caractère déterminant, pour leurs activités quotidiennes de contrôle, des indices communiqués par les populations locales, sans lesquels «le travail de la police ne serait même pas possible 10 ».

En République tchèque, au contraire, étant donné que le contrôle de la frontière avait été assuré par l'armée pendant plus de quarante ans dans la Tchécoslovaquie socialiste, le transfert du contrôle frontalier à l'administration policière civile constitue l'une des premières missions du nouveau gouvernement non communiste mis en place après la chute du régime. Si les années de communisme se caractérisaient, comme dans l'ensemble des pays sous influence soviétique, par une visibilité importante du contrôle frontalier et une certaine héroïsation du garde-frontière ${ }^{11}$, il semble que le contrôle frontalier soit devenu, après la Révolution de velours, un élément relativement silencieux de la politique nationale de sécurisation du territoire. D’abord, la récente ouverture des frontières paraît rendre peu souhaitable le rétablissement de contrôles frontaliers très visibles et susceptibles de rappeler aux habitants le régime précédent. Ensuite, la Tchécoslovaquie (puis la République tchèque) adopte alors une politique très libérale d'accueil des immigrés et des réfugiés (encore relativement peu nombreux) qui n'impose pas de contrôle très strict des frontières nationa-

8. Voir la campagne d'information lancée à l'automne 1999 par l'armée autrichienne dans la presse nationale, citée dans Fronek H., «Illegalisierung in Österreich » [Illégalisation en Autriche], SWS-Rundschau, n 1,2000 , pp. 89-99. Notre traduction.

9. Ibid., p. 91.

10. Les citations insérées dans le texte sont extraites, sauf mention contraire, d'observations et d'entretiens conduits avec des fonctionnaires de la police des étrangers et des frontières tchèque (Cizinecká a pobranicní policie) et de la police des frontières autrichienne (Grenzkontrollwesen) dans différents postes frontières tchèques et autrichiens entre 2004 et 2007 et ont été traduites par l'auteur. Bien que l'entrée de la République tchèque dans l'Union européenne en mai 2004 ait conduit à la suppression des formalités douanières, et par conséquent à la disparition des bâtiments et du corps professionnel des douaniers, les policiers interrogés étaient en charge, outre du contrôle migratoire, de la détection des infractions aux réglementations limitant le transport de cigarettes notamment. Nous désignerons ici nos interlocuteurs par l'appellation générique « policiers des frontières » ou « gardes-frontières », la plus proche des désignations courantes en tchèque et en allemand.

11. Bien que le contrôle des frontières sous le communisme semble avoir jusqu'alors été relativement peu traité par l'historiographie tchèque, nous renvoyons, pour une étude du contrôle frontalier sous régime communiste, à l'ouvrage : Coeuré S., Dullin S. (dirs.), Frontières du communisme, Paris, La Découverte, 2007. 
les. C'est seulement sous la pression des pays voisins (Allemagne et Autriche), puis de l'Union européenne ${ }^{12}$, que la République tchèque fait du contrôle frontalier un espace de démonstration de sa capacité à assurer la sécurité de son territoire et, surtout, des frontières de l'espace Schengen.

Cette politique d'affichage ne semble cependant pas faire l'objet, sur la scène nationale et locale, d'investissements communicationnels ou symboliques du même ordre que ceux observés en Autriche depuis le début des années 1990. Les policiers tchèques décrivent ainsi les indications reçues de la population comme un élément très marginal du contrôle frontalier et attribuent la faible propension des populations frontalières de République tchèque à signaler les personnes suspectées d'immigration clandestine à une "différence de mentalité » entre Autrichiens et Tchèques, ces derniers n'étant "pas encore éduqués » pour aider la police à identifier les individus «suspects». L'histoire des frontières et de leur surveillance dans la Tchécoslovaquie communiste explique sans doute, en partie du moins, la réticence des habitants à s'associer au contrôle des espaces frontaliers : sous le régime communiste, la population locale a en effet souvent joué, par le biais des patrouilles civiles de "gardes auxiliaires 》(Pomocnípohranicní stráz), un rôle au moins aussi important que l'armée elle-même dans le contrôle des frontières nationales ${ }^{13}$. Depuis l'ouverture des frontières, il semble donc que la participation des populations locales au contrôle frontalier soit associée aux pratiques délatrices en cours sous le régime communiste, et majoritairement perçue de manière négative par les habitants des régions frontalières.

Peu médiatisé, le contrôle des frontières de la République tchèque n'en reste pas moins central dans le rapport du pays aux instances européennes, notamment à partir de 1998 et du dépôt de sa candidature à l'entrée dans l'Union: les frontières physiques et aéroportuaires constituent en effet les lieux privilégiés d'évaluation, par les instances européennes, de la capacité de la République tchèque (comme des autres Etats alors candidats) à intégrer l'UE dans un premier temps, puis l'espace Schengen.

12. Le programme PHARE d'aide communautaire aux pays d'Europe centrale et orientale, lancé en 1994 pour apporter un soutien financier à la stratégie de pré-adhésion des Etats candidats à l'entrée dans l'Union européenne, joue ici un rôle déterminant : reprenant certaines initiatives bilatérales, en particulier l'allocation par l'Allemagne de fonds importants à la République tchèque et à la Pologne en 1993 afin d'assister ces pays dans le développement d'outils de contrôle des frontières plus efficaces, le programme PHARE consacre une part importante de son budget annuel aux questions relatives à la justice et aux affaires intérieures, notamment au développement de la coopération transfrontalière et à l'amélioration technique de la surveillance des frontières extérieures. Voir Guiraudon V., "The Constitution of a European Immigration Policy Domain: A Political Sociology Approach", Journal of European Public Policy, vol. 10, n², 2003, pp. 263-282 (pp. 271-272). En 2001, 163 millions d'euros ont été consacrés à la coopération transfrontalière, soit environ $10 \%$ du budget total annuel du programme PHARE.

13. Sur ce point, voir notamment la communication de Blaive M., "Mémoires du Rideau de fer, mémoires du communisme : Ceské Velenice, une ville tchèque à la frontière de l'Autriche " au colloque «Présence du passé. Mémoires et sociétés du monde contemporain », Musée du Quai Branly, 30 novembre 2007. 


\section{La frontière Schengen : lieu de passage et d'ajustement des straté- gies migratoires et policières}

C'est en 2004, année de l'adhésion de la République tchèque à l'UE, que semble se cristalliser le rôle particulier de la frontière entre l'Autriche et la République tchèque en matière d'immigration irrégulière. Alors que, de 1993 à 2003, la plupart des migrants interpellés en République tchèque pour franchissement illégal de la frontière l'avaient été à la frontière tchéco-allemande, après être arrivés en République tchèque depuis la Slovaquie, en 2004, 43,5 \% des 10695 personnes interpellées pour migration illégale à la frontière le sont à la frontière tchéco-autrichienne (longue de 443 kilomètres), contre seulement $23 \%$ à la frontière avec l'Allemagne ${ }^{14}$, pourtant deux fois plus longue (815 kilomètres). Cet accroissement de la pression migratoire sur la frontière tchéco-autrichienne est généralement imputé à l'augmentation des flux de migrants tchétchènes arrivant de Pologne en République tchèque et cherchant à gagner l'Autriche pour y déposer une demande d'asile : au cours des quatre premiers mois de l'année 2004 - et alors que la République tchèque n'est pas encore membre de l'UE, - la police enregistre en effet une forte hausse du nombre de migrants russes (dont la plupart est originaire de Tchétchénie) interceptés alors qu'ils tentent de franchir illégalement la frontière qui sépare la République tchèque de l'Autriche. L'Autriche constituant pour la majeure partie d'entre eux le pays de destination finale, il semble que les agents facilitateurs de l'entreprise migratoire aient cherché à faire passer le plus grand nombre possible de migrants tchétchènes par la République tchèque avant l'entrée de cette dernière dans l'Union européenne. Jusqu'au 1er mai 2004 en effet, la plupart d'entre eux avait transité par les centres pour demandeurs d'asile de Pologne, où ils avaient déposé une première demande. En cas d'interception en République tchèque, ou si une étape était nécessaire à l'organisation de leur passage vers l'Autriche, ils déposaient une deuxième demande auprès des autorités tchèques, et enfin une troisième après leur arrivée à destination en Autriche. Ainsi, durant les trois premiers mois de l'année 2004, 70 \% des migrants de nationalité russe arrêtés à la frontière tchèque alors qu'ils tentaient de quitter le pays avaient le statut de demandeurs d'asile en République tchèque ${ }^{15}$. Cependant, l'entrée en vigueur, en République tchèque et en Pologne, du Règlement Dublin II 16 à partir de mai 2004 permet désormais de renvoyer vers ces deux pays les demandeurs d'asile dont il est établi qu'ils y ont déposé leur première demande de protection. La stratégie migratoire des demandeurs d'asile tchétchènes a donc évolué : il semble d'abord qu'ils aient

14. Voir ministère de l'Intérieur de la République tchèque, 2004 Status Report on Migration in the Czech Republic, Prague, MVCR, 2005, pp. 94-96.

15. Ibid., p. 92.

16. Le Règlement n`343/2003 du Conseil du 18 février 2003 (ou Règlement Dublin II), qui a remplacé en 2003 la Convention de Dublin signée en 1990, établit les critères et mécanismes de détermination de l'Etat membre responsable de l'examen d'une demande d'asile présentée dans l'un des Etats membres par un ressortissant d'un pays tiers. 
davantage cherché à éviter le contact avec les autorités tchèques (qui ont enregistré une baisse brutale du nombre de migrants tchétchènes interceptés à la frontière au cours de la seconde moitié de l'année 2004), afin que leur passage par le territoire de la République tchèque ne puisse être documenté. En outre, et sans doute pour cette même raison, le recours aux passeurs pour le franchissement de la frontière tchéco-autrichienne a considérablement augmenté dans les statistiques policières en 2004, les citoyens russes représentant alors près de la moitié des personnes venues avec l'aide d'un passeur ${ }^{17}$.

L'évolution des stratégies migratoires induite par l'entrée de la République tchèque dans l'UE est également perceptible à travers le constat suivant : au premier semestre 2004, plus de la moitié des interpellations à la frontière concernaient des migrants, tchétchènes pour la plupart (et, dans une moindre mesure, géorgiens et ukrainiens) dont l'entrée et le séjour était légaux (en raison du statut de demandeur d'asile dont la plupart d'entre eux bénéficiaient en République tchèque), mais qui tentaient de sortir illégalement du territoire (la sortie du territoire n'étant pas autorisée aux demandeurs d'asile). Au contraire, la seconde moitié de l'année 2004 (après l'adhésion de la République tchèque à l'UE) et l'année 2005 voient une forte augmentation des arrestations de migrants entrés illégalement sur le territoire tchèque, confirmant les tentatives d'évitement du contact avec les autorités nationales tchèques jusqu'à l'arrivée à destination (en l'occurrence, l'Autriche) ${ }^{18}$. L'entrée de la République tchèque dans l'UE a un autre effet notoire sur les modes de franchissement illégal des frontières nationales, en conduisant à une utilisation accrue, à compter du milieu de l'année 2004, de faux documents : l'intégration de la Tchéquie à l'UE allège en effet les contrôles effectués sur les documents d'identité de citoyens européens, ce qui encourage un recours plus fréquent aux documents des nouveaux pays membres dont la falsification est la plus aisée, et notamment aux faux documents slovaques, polonais et lituaniens ${ }^{19}$.

Par ailleurs, depuis l'entrée de la République tchèque dans l'espace Schengen le 21 décembre 2007, l'augmentation en Autriche du nombre de demandeurs d'asile originaires de Tchétchénie et ayant transité par la République tchèque (souvent depuis la Pologne) est venue replacer la frontière, désormais ouverte, au cœur des relations bilatérales tchéco-autrichiennes. Les autorités autrichiennes semblent en effet tenir la police tchèque pour responsable du doublement, quelques jours après l'ouverture de l'ancienne frontière Schengen, des demandes

17. Ministère de l'Intérieur de la République tchèque, 2004 Status Report on Migration in the Czech Republic, op. cit., p. 93 et p. 109.

18. Ainsi, les demandeurs d'asile représentaient $22 \%$ des étrangers interceptés lors du franchissement illégal d'une frontière nationale en 2004 , mais à peine $5 \%$ en 2005 . Voir : ministère de l'Intérieur de la République tchèque, 2004 Status Report on Migration, op. cit., pp. 125-126; 2005 Status Report on Migration in the Czech Republic, Prague, MVÈR, 2006, p. 144.

19. Voir ministère de l'Intérieur de la République tchèque, 2004 Status Report on Migration, op. cit., p. 105. 
d'asile enregistrées dans le principal centre de réception de Traiskirchen (BasseAutriche) et émanant pour la plupart de citoyens russes (supposés originaires de Tchétchénie) ayant auparavant déposé une demande dans un autre pays membre, en général la Pologne ou la République tchèque ${ }^{20}$.

Si les chiffres collectés aux frontières par les polices tchèque et autrichienne peuvent témoigner du potentiel de réactivité des migrants et de leurs passeurs face à l'évolution des contrôles, ils rappellent également le caractère incertain des statistiques policières. Il reste en effet difficile de déterminer dans quelle mesure la baisse relative de l'immigration irrégulière enregistrée dans les deux pays depuis 2004 est imputable à un ciblage accru des contrôles, aux évolutions législatives ou à une plus grande efficacité de l'autorité policière, ou s'il faut au contraire y voir le résultat d'un recours accru des migrants et de leurs passeurs à des stratégies et des itinéraires dont la détection est aléatoire et difficile, tel par exemple le transit par camions qui enregistre une forte hausse. Les aléas entourant la collecte de données statistiques relatives à un phénomène par définition occulte, telle l'immigration irrégulière, incitent par conséquent à manipuler avec prudence les chiffres disponibles, qui ne peuvent jamais s'appuyer que sur les cas de migration illégale documentés par les autorités, c'est-à-dire ceux où l'illégalité entre en contact avec l'administration étatique ${ }^{21}$. S'ils informent sur certaines grandes tendances, tant du point de vue des migrations que de celui de leur contrôle, les chiffres relatifs au franchissement illégal de la frontière ne sauraient fonder à eux seuls l'analyse, et ne servent ici qu'à contextualiser la portion de frontière ethnographiée, entre la République tchèque et l'Autriche.

\section{Le contrôle frontalier en pratiques}

Les enquêtes de terrain réalisées dans les postes frontières révèlent tout d'abord les nombreux aléas auxquels est soumis le contrôle des flux migratoires aux frontières nationales, présenté par le ministère de l'Intérieur comme une priorité absolue de l'Autriche, et dont la police tchèque a, quant à elle, dû démontrer le caractère implacable pour se voir reconnaître le droit d'entrer dans l'UE (et, plus récemment, dans l'espace Schengen). Ces variations, qui

20. Le ministre de l'Intérieur autrichien a ainsi indiqué, dès le début du mois de janvier 2008, que plus de la moitié des personnes ayant déposé une demande d'asile en Autriche au cours des derniers jours (soit depuis l'entrée dans l'espace Schengen, le 21 décembre 2007, des pays voisins de l'Autriche ayant adhéré à l'UE en 2004) étaient déjà enregistrés dans le système EURODAC. Cette inscription signifie soit qu'ils avaient déjà déposé une demande d'asile auprès d'un autre Etat membre de l'UE, soit qu'ils avait été interceptés en situation irrégulière par un autre Etat membre et, qu'en tous les cas, l'Autriche n'était donc pas responsable du traitement de leur demande d'asile. Voir «Verfassungsexperte skeptisch bei Heer-PolizeiKooperation » [Les experts constitutionnels sceptiques quant à la coopération armée-police], Der Standard, 7 janvier 2008.

21. Signalons par ailleurs la tendance des statistiques policières à comptabiliser non les migrants mais les interpellations, conduisant ainsi à l'enregistrement répété du même migrant interpellé à différentes reprises pour entrée irrégulière sur le territoire. 
sont essentiellement fonction du moment de la journée, de la nationalité de la personne franchissant la frontière ou encore de l'employé de police affecté au contrôle, questionnent donc la représentation de la frontière comme un espace de contrôle technicisé, réduisant à son minimum le poids du hasard et font au contraire apparaître une certaine malléabilité des pratiques de contrôle, comme le confirme cette citation d'un policier des frontières autrichien :

«Bien sûr, c'est un hasard si un migrant illégal passe justement par là au moment où nous contrôlons. »

[Extrait du journal de terrain, frontière autrichienne, septembre 2006]

\section{Les aléas du contrôle : variations horaires et saisonnières}

Les contrôles des personnes et des véhicules franchissant la frontière sont plus stricts la nuit que le jour, et les policiers confirment le caractère a priori plus suspect des voyageurs nocturnes en affirmant que «les passages illégaux ont quasiment toujours lieu la nuit ». En effet, le trafic diurne est considéré comme majoritairement constitué de travailleurs (transfrontaliers notamment), le franchissement nocturne étant au contraire le propre de «ceux qui ont quelque chose à cacher». En outre, le fait que le personnel policier des postes frontières soit nécessairement moins sollicité de nuit, en raison du caractère réduit du trafic, explique également que les contrôles y soient souvent conduits avec plus de soin et d'attention. La « frontière verte 22 » fait elle aussi l'objet d'une surveillance nocturne plus rigoureuse, que les policiers justifient non seulement par la fréquence statistiquement plus élevée des passages clandestins la nuit, mais aussi par le fait que la vigilance des populations locales limite, du côté autrichien, les possibilités de franchissement illégal de la frontière verte (par les champs, les bois ou les vignes) en journée. Ainsi, alors qu'elle n'est surveillée de jour que sporadiquement par quelques patrouilles en voiture, la frontière verte entre la République tchèque et l'Autriche est soumise de nuit à un contrôle accru, reposant notamment sur l'utilisation de caméras thermo-vision. Disposées à certains endroits stratégiques surplombant une importante portion de la frontière verte, celles-ci permettent de détecter toute source de chaleur, et partant toute présence humaine, dans un rayon de vingt kilomètres environ par temps clair.

Johnny ${ }^{23}$, ancien policier de nationalité tchèque et ayant participé à l'organisation du passage clandestin de près de 150 personnes depuis la République tchèque vers l'Italie (via l'Autriche), confirme le caractère fluctuant du

22. On désigne par «frontière verte » la portion des frontières nationales située en pleine nature entre deux postes frontières.

23. Le prénom a été modifié par l'auteur. Les citations de Johnny sont extraites de l'entretien conduit à Prague le 25 septembre 2007. 
contrôle policier aux frontières, qui présente selon lui « des cycles ». Il décrit le passage de migrants clandestins en journée comme beaucoup plus risqué car les policiers auraient alors «trop de temps », du moins en dehors des pics d'activité correspondant aux horaires d'embauche et de sortie du bureau, et se livreraient donc à un contrôle plus minutieux du véhicule et de ses occupants. Le moment qui présenterait, selon Johnny, le moins de risques pour le passage clandestin se situe la nuit, entre $3 \mathrm{~h}$ et $6 \mathrm{~h}$ du matin, au moment où, après une phase d'activité intense entre $1 \mathrm{~h} 30$ et $3 \mathrm{~h}$ du matin, un grand nombre de policiers se reposent et l'effectif policier est donc le plus réduit. Mais les risques liés au franchissement illégal de la frontière semblent également différer d'un jour à l'autre de la semaine : Johnny présente les fins de semaine comme "risquées ", la présence de nombreux touristes contraignant la police à des contrôles d'identité plus poussés qu'en semaine, où les travailleurs frontaliers constituent la majeure partie du trafic. Le milieu de semaine se prêterait au contraire particulièrement bien aux opérations de passage clandestin de migrants en raison de l'important trafic de camions alors enregistré : celui-ci rend non seulement les contrôles des véhicules particuliers plus aléatoires, la plupart des efforts se concentrant sur les camions et leur marchandise, mais il permet également aux passeurs de «coller » leur véhicule derrière un camion dans la zone précédant la frontière et placée sous surveillance vidéo. Le véhicule du passeur est alors dissimulé, sur l'écran de contrôle auxquelles sont reliées les caméras qui jalonnent les routes d'accès au poste frontière, par le camion qui le devance et n’éveille ainsi pas l'attention des policiers.

Les saisons déterminent également le risque associé au passage clandestin de la frontière : la période estivale est ainsi unanimement décrite, par les policiers mais aussi par les passeurs et les migrants interrogés, comme la plus propice au franchissement clandestin et piéton de la frontière verte. Avant les moissons, les champs de blé offrent en effet une protection idéale aux migrants et, jusqu'aux premiers jours de l'automne, les champs de tournesol non coupés rendent la présence d'étrangers difficilement repérable par les patrouilles et caméras de contrôle. Au contraire, la fin de l'automne et l'hiver, marqués par une végétation rare et des conditions climatiques potentiellement difficiles, présentent des risques accrus pour les migrants tentant de franchir à pied la frontière verte.

\section{Définition $d u$ «suspect » : les logiques de tri}

D'autres facteurs peuvent cependant également influer sur la décision des policiers d'appliquer des formes plus ou moins strictes de contrôles. Ainsi, conformément aux réglementations européennes prévoyant des modalités de contrôles allégées pour les citoyens de l'UE aux frontières de l'espace Schengen, telle la frontière tchéco-autrichienne entre 2004 et 2007, les ressortissants tchèques ou autrichiens (de même que la plupart des citoyens des autres pays membres de l'UE) se présentant au poste frontière que nous avons ethnographié ne sont généralement contrôlés que très sommairement : le poli- 
cier se contente d'un coup d'œil rapide, depuis la guérite où il est installé, sur la pièce d'identité qu'on lui présente, voire simplement sur la couverture de celle-ci, sans vérifier ni l'inscription du voyageur dans un fichier sécurisé, national ou européen, ni le contenu du véhicule. Johnny présente lui aussi le fait d'être citoyen tchèque et de circuler dans un véhicule immatriculé en République tchèque comme un avantage pour le déroulement des opérations de passages clandestins de migrants auxquelles il a participé : en conversant de manière anodine et en tchèque avec les policiers des frontières tchèques, il pense avoir pu échapper au stigmate qui fait apparaître l'étranger comme a priori suspect, et qui explique selon lui que «les passeurs ukrainiens par exemple se $f$ [assent $]$ plus facilement repérer à la frontière ».

Dans un contexte de (quasi) libre circulation des citoyens européens, un contrôle approfondi est pourtant parfois pratiqué, quand bien même le voyageur présente un document d'identité européen et que son véhicule est immatriculé en Europe : les policiers des frontières justifient avant tout ces contrôles, de nature plutôt exceptionnelle, par l'«allure louche » ou l'« air suspect » du voyageur. Bien que les caractéristiques permettant de déterminer le degré de suspicion qu'éveille un voyageur soient difficiles à recenser, les policiers déclarant généralement faire confiance à leur « instinct », certains critères semblent, au fil des journées d'observation dans le poste frontière, guider la décision des gardes-frontières : ainsi, les hommes sont ordinairement plus contrôlés que les femmes, les personnes seules plus que les couples et les jeunes plus que les voyageurs plus âgés. Par ailleurs, les véhicules de type camionnettes, ou encore les voitures accompagnées d'une caravane, font l'objet d'un contrôle quasi systématique, de même que les représentants de certaines nationalités, attestant l'hypothèse d'une ethnicisation (voire d'une racialisation) du contrôle : outre les voyageurs présentant des signes extérieurs d'altérité (liés à la couleur de peau notamment), les visiteurs munis de documents italiens et, plus encore, polonais, se voient également soumis à un contrôle minutieux quasi systématique résultant de l'anticipation, par les policiers, de comportements-types de la part des populations contrôlées. Les voyageurs munis de papiers italiens sont ainsi suspectés d'usage de faux documents et/ou d'implication dans le passage de migrants clandestins, l'itinéraire reliant la République tchèque à l'Italie constituant actuellement l'un des trajets les plus empruntés par les migrants en situation irrégulière et leurs passeurs. La présentation de papiers polonais active, quant à elle, dans les représentations policières, le soupçon de vol, et éventuellement de trafic de migrants, notamment lorsque les voyageurs circulent en camionnette (ce qui se trouve être fréquemment le cas).

\section{Le "flair» du policier : prestige $d u$ "chien de chasse »}

Enfin, un autre paramètre paraît jouer un rôle déterminant dans la précision et l'intensité du contrôle, à savoir le choix du policier affecté au contrôle. Au sein même de l'institution, les gardes-frontières opèrent une distinction 
entre les collègues présentés comme des «chiens de chasse » (Schnüffelbund) et les autres. Les premiers, décrits comme des "génies du contrôle », reçoivent en général cette étiquette après s'être illustrés par la détection d'importantes illégalités à la frontière, et leur "génie » trouve à s'exprimer dans différents domaines de spécialité : l'un sera présenté comme le «champion de la détection des illégaux », un autre comme la terreur des conducteurs de poids lourds ne respectant pas la législation en matière de licence ou de poids des marchandises, un troisième, enfin, comme expert du trafic de cigarettes. Ces employés modèles se livrent à un contrôle plus précis et rigoureux que leurs collègues, tout en justifiant également leur choix des véhicules et des personnes à contrôler par le recours à un "sixième sens ", leur "instinct» leur permettant de «sentir» les cas suspects.

L'observation des pratiques locales de contrôle renseigne également sur le fonctionnement interne de l'institution policière et sur les échelles de valorisation qui s'y développent. Alors que les responsables politiques et policiers rencontrés à Vienne et Prague au sein de la direction générale de la police ou du ministère de l'Intérieur s'attachent à présenter les frontières comme un espace sous contrôle, au sein duquel les investissements technologiques et humains ne laissent aucune place au hasard, il semble que la situation géographique à la marge des espaces frontaliers autorise leurs acteurs à se distancier des représentations centrales de la frontière comme lieu du contrôle mécanique et figé :

«Tout est politique. La sécurité est un argument de vente de la politique. La politique se sent obligée d'investir de l'argent dans la sécurité. Peu importe que ça fonctionne ou non, ou que les policiers euxmêmes disent que ça ne sert à rien. [...] En fait, nous ne faisons plus qu'administrer le problème, nous ne le contrôlons pas. Les migrations ne peuvent de toute façon pas être arrêtées ou combattues à la frontière, là il est déjà trop tard ».

[Entretien conduit avec un représentant du ministère de l'Intérieur autrichien en déplacement à la frontière autrichienne, septembre 2006]

De fait, le contrôle frontalier apparaît, en dépit des politiques nationales de mise en visibilité de la frontière, comme relativement peu prestigieux dans les perceptions des policiers eux-mêmes, en raison notamment de sa relégation géographique aux confins du territoire. La plupart des gardes-frontières sont donc soit eux-mêmes originaires des régions frontalières, où ils ont été directement affectés après leur entrée dans la police, sans être passés par d'autres services ou régions, soit de jeunes recrues qui, pour leur première année d'exercice, n'ont pas eu le choix de leur affectation et ont donc été envoyées dans les postes frontières. Pour ces dernières, qui vivent généralement mal l'éloignement de leur région d'origine et de leur univers social et affectif, le contrôle frontalier est un lieu de relégation non seulement géographique, mais aussi pro- 
fessionnelle, l'activité y étant présentée comme majoritairement routinière. Car si elles sont volontiers relatées par les policiers eux-mêmes, les interpellations de migrants en situation irrégulière et/ou de passeurs restent rares, ce qui explique d'ailleurs qu'elles s'ancrent d'autant mieux dans la mémoire des policiers et que leur souvenir soit fréquemment réactivé. La plupart du temps, les policiers des frontières sont pourtant occupés à des tâches moins prestigieuses, dans l'échelle de valeurs interne à l'organisation policière, que le débusquage d'un passage clandestin, et notamment à la rédaction de contraventions à l'intention des poids lourds ne respectant pas la réglementation ou, plus modestement encore, au contrôle routinier de voyageurs auxquels n'est associé aucun soupçon de déviance potentielle, et dont les documents sont donc à peine regardés. Ce qui semble faire le prestige particulier des arrestations présentées comme le fruit du «flair» du policier, c'est donc d'abord leur caractère valorisant pour l'ensemble de la profession, dont la légitimité est avant tout construite par les discours de sécurité nationaux sur le contrôle et la répression de l'immigration irrégulière et de la criminalité transfrontalière.

\section{La coopération policière transnationale}

Quand bien même la hiérarchie policière n'a de cesse de rappeler le rôle essentiel dévolu à la coopération transnationale dans la lutte contre l'immigration non documentée, les observations conduites illustrent le décalage - également observé dans d'autres contextes de recherche ${ }^{24}$ - entre la création, au niveau national, des conditions structurelles de la coopération, et les motifs opérationnels des forces de police locales à coopérer.

Ainsi, le centre tchéco-autrichien de coopération policière, inauguré à l'automne 2006 et destiné à renforcer la sécurité aux frontières étatiques communes à travers le développement de la coopération (notamment par le biais de patrouilles binationales) dans la lutte contre l'immigration irrégulière ou la criminalité transfrontalière, est prévu pour accueillir un total de vingt-quatre fonctionnaires de police (douze Tchèques et douze Autrichiens, tous supposés bilingues). Malgré la médiatisation importante dont a bénéficié l'inauguration du centre, celui-ci se résume, dans les premiers mois de son activité, à un espace en travaux, où errent quelques policiers désœuvrés qui peinent à trouver une langue commune. Six mois après leur lancement, les patrouilles communes 25 le long de la frontière verte, présentées comme l'un des piliers de la coopération transfrontalière, ont lieu une à deux fois par mois. Bien qu'en principe composées de policiers capables de s'exprimer dans la langue du territoire sur lequel est conduite l'opération, elles réunissent le plus souvent des

24. Voir par exemple Deflem M., "Bureaucratization and Social Control: Historical Foundations of International Police Cooperation”, Law E Society Review, vol. 34, n³, 2000, pp. 739-778.

25 . Les patrouilles communes réunissent deux policiers autrichiens et un policier tchèque en territoire autrichien, et deux policiers tchèques et un policier autrichien en territoire tchèque. 
policiers autrichiens non tchécophones et des policiers tchèques non systématiquement germanophones. Il arrive donc que les policiers en patrouille ne disposent d'aucune langue commune. En outre, les patrouilles binationales ne sont déployées qu'en journée, à des moments où l'activité policière de lutte contre l'immigration non documentée est quasi nulle, et sont présentées par les policiers eux-mêmes comme destinées avant tout à «faire connaissance ». Elles alternent ainsi échanges linguistiques, commérages locaux ainsi que quelques opérations ponctuelles de contrôle de la route pratiquées, dans un relatif flou juridique, sur des routes peu passagères et fréquentées essentiellement par des travailleurs frontaliers :

«En fait, personne ne sait encore vraiment quels sont nos droits et nos devoirs. Par exemple, on ne sait pas s'il est prévu que nous fassions des contrôles routiers, ni quels seraient nos droits et ceux de nos collègues tchèques s'il se produisait quelque chose [c'est-à-dire une arrestation] dans le cadre d'une patrouille commune. »

[Extrait du journal de terrain, frontière tchéco-autrichienne, juin 2007]

Lieu de cohabitation et de prise de contact entre les représentants des deux polices nationales, le centre de coopération est également celui du face-à-face, de la comparaison, voire de la confrontation entre deux administrations : tandis qu'ils arpentent la frontière verte dans de puissants et coûteux véhicules récemment acquis, les policiers autrichiens, qui s'estiment généralement "très bien payés", décrivent avec condescendance la vétusté des véhicules de la police tchèque, leur rationnement en essence et les salaires de leurs collègues tchèques, «trois fois inférieurs » aux leurs.

La coopération policière transnationale, érigée en priorité de la lutte contre l'immigration irrégulière par les discours de la hiérarchie policière nationale et des instances européennes, voit donc se dessiner sur le terrain des pratiques plus aléatoires et des discours plus réservés. A la frontière tchécoautrichienne, les modalités de coopération locale semblent sous-tendues par la conviction d'une supériorité de la police autrichienne sur son homologue tchèque. De fait, la langue de la coopération reste l'allemand (en dépit des discours officiels sur l'objectif de bilinguisme des policiers), l'échange d'information entre les polices est réduit et les gardes-frontières autrichiens rencontrés s'appliquent à conserver, dans leur rapport à la police tchèque, le rôle confortable de «donneurs de leçons », qu'ils justifient notamment par des ressources matérielles et financières plus importantes.

Il convient toutefois de garder à l'esprit le contexte de tensions particulières, liées à l'élargissement programmé de l'espace Schengen (effectif depuis décembre 2007), dans lequel les enquêtes sur lesquelles nous appuyons nos analyses ont été réalisées : alors que les policiers des frontières doivent au contrôle frontalier leur implantation professionnelle dans les régions frontalières, l'an- 
nonce de la disparition des postes frontières tchéco-autrichiens a souvent été interprétée non seulement comme une remise en cause de leur légitimité socioprofessionnelle, mais aussi comme synonyme d'une grande incertitude de carrière. Ce sentiment a pu être attisé par la position relativement trouble tenue, peu de temps encore avant l'élargissement de l'espace Schengen, par la hiérarchie policière quant au devenir des policiers affectés au contrôle des frontières appelées à disparaître : les policiers tchèques, minés par le contexte général difficile de leur institution ${ }^{26}$, n'ont d'abord reçu aucune garantie concernant leur avenir professionnel et ignoraient toujours, quelques mois avant la date prévue pour l'élargissement, si leur affectation dans les régions frontalières resterait maintenue après l'entrée de la République tchèque dans Schengen. Les gardesfrontières autrichiens, quoique assurés de leur côté de conserver leur emploi en région frontalière par le biais d'un redéploiement du contrôle sur l'ensemble de la zone frontalière afin de compenser la suppression des postes frontières ${ }^{27}$, considéraient malgré tout la disparition programmée de la frontière et de son contrôle comme une menace. En marge des discours de la hiérarchie, s'attachant à mettre en avant les bienfaits de la coopération transfrontalière et les progrès réalisés par les pays voisins dans le contrôle des flux de personnes, les policiers des frontières autrichiens rencontrés rappelaient au contraire régulièrement les insuffisances de la police tchèque et le déficit de sécurité qui ne manquerait pas, à leurs yeux, de découler de l'élargissement de l'espace Schengen. La défiance témoignée à l'égard de l'abolition des contrôles aux postes frontières semble participer d'une tentative des gardes-frontières de re-légitimer leur fonction, que la disparition du contrôle frontalier menace a priori. Pour ce faire, il leur appartient en effet d'attirer l'attention de l'interlocuteur sur les dangers qui accompagneront nécessairement la réduction des contrôles et que seule leur présence aux frontières permettait jusqu'alors de contenir. L'élargissement de l'espace Schengen n'ayant pas pour effet d'abolir les contrôles mais, tout au plus, d'en déplacer la focale vers les frontières Schengen extérieures, cet argumentaire doit s'appuyer sur une critique des capacités de contrôle des pays qui s'en partagent désormais la charge. Bien que les frontières de la République tchèque ne coïncident pas avec les frontières extérieures de l'espace Schengen, le fait qu'elle soit un pays limitrophe de l'Autriche fait peser sur elle, aux yeux des policiers autrichiens, une part importante de la responsabilité du contrôle des flux à destination de l'Autriche et, partant, de la sécurité du territoire autrichien. La rhé-

26. Voir notamment les difficultés rencontrées par la police tchèque pour recruter de nouveaux fonctionnaires et couvrir ses besoins en personnel, évoquées dans : Darley M.A., Frontière, asile et détention des étrangers. Le contrôle étatique de l'immigration et son contournement, op. cit., p. 279.

27. De fait, depuis l'entrée de la République tchèque dans l'espace Schengen, la police autrichienne a fortement intensifié les contrôles conduits sur le territoire autrichien, à quelques kilomètres seulement des anciens postes frontières. Voir les articles parus notamment dans la presse tchèque en janvier 2008, par exemple " "Schengen Kontrolle”, slyší Ceši dál v Rakousku " [ "Contrôle Schengen », voilà ce que les Tchèques continuent d'entendre en Autriche], Lidové Noviny, 18 janvier 2008, ou «Chceme mít prehled » [Nous voulons avoir une vue d'ensemble], Lidové Noviny, 18 janvier 2008. 
torique déployée par les gardes-frontières, soucieux de rappeler à tout intervenant extérieur le caractère indispensable de la fonction autour de laquelle s'est jusqu'alors articulé l'ensemble de leur légitimité sociale et professionnelle, consiste donc à discréditer les capacités de contrôle des policiers des frontières tchèques en insistant non seulement sur la faiblesse des équipements et des ressources humaines, mais aussi sur leur «retard éthique » supposé, qui expliquerait leur plus grande propension à la corruption.

\section{Le déplacement de la frontière Schengen : vers une nouvelle géo- graphie du contrôle?}

La frontière, outre sa centralité dans les politiques nationales et européennes de lutte contre l'immigration irrégulière, apparaît donc ici non pas tant comme un espace d'évaluation du contrôle que comme le lieu d'un face-à-face, dans un contexte d'incertitude lié à la l'élargissement programmé de l'espace Schengen, entre deux institutions policières. Une ethnographie des pratiques locales de coopération révèle en effet, en marge des discours nationaux et européens vantant les mérites du rapprochement policier transfrontalier, la matérialité que conserve la frontière dans les représentations policières locales. Elle semble ainsi avant tout envisagée par les policiers autrichiens comme le lieu de la réaffirmation de leur supériorité (financière, matérielle, linguistique ou "éthique ») sur l'institution policière tchèque. On peut poser l'hypothèse que les formes «molles » revêtues par la coopération transfrontalière tchéco-autrichienne résultent de fait de la non-émergence d'un «ennemi commun 28 », pour les deux polices nationales, en la figure du « migrant clandestin » en raison notamment des perceptions différenciées qui accompagnent, sur chacun des territoires nationaux, les régions frontalières et leur importance géopolitique et stratégique.

Ces divergences tiennent d'abord à la nature même de la frontière qui, si elle peut être présentée par la police et les autorités autrichiennes comme un rempart contre l'infiltration du « crime » de l'Est, constitue au contraire, dans le contexte tchèque, une porte de sortie plus qu'une porte d'entrée pour les flux clandestins de personnes, dont la direction principale reste d'Est en Ouest. Dès lors, malgré l'engagement pris depuis plusieurs années par la République tchèque de contribuer à la protection de l'espace Schengen, et bien que le contrôle frontalier fasse ici office de vitrine des efforts déployés, la charge émotionnelle potentiellement associée au contrôle frontalier se trouve nécessairement amoindrie par la position de la frontière observée sur le territoire tchèque. A ces considérations géographiques s'ajoutent pourtant des facteurs historiques et situationnels dont la portée explicative semble plus profonde : bien que géographiquement périphérique, la frontière est, en Autriche, au cour du dispositif de contrôle migratoire et fait l'objet d'investissements

28. Deflem M., “Bureaucratization and Social Control...”, op. cit., p. 746. 
rhétoriques, matériels et humains importants de la part de l'institution policière et des autorités politiques. Au contraire, en République tchèque, la situation aux confins des zones frontalières semble redoublée par leur perception, tant par les populations locales que par les représentants politiques et policiers nationaux, comme des espaces de relégation ${ }^{29}$.

La disparition, en décembre 2007, de la frontière Schengen séparant jusqu'alors l'Autriche de la République tchèque a cependant entraîné des restructurations conséquentes du contrôle frontalier : les postes frontières sur lesquels avait porté notre étude ont disparu, tandis que le centre tchéco-autrichien de coopération policière est appelé à jouer un rôle de plus en plus important. Les contrôles, qui ne peuvent désormais plus être effectués sur le tracé de la limite nationale, ont fait l'objet d'un redéploiement sur le territoire. Ainsi, en Autriche, la police des frontières a le plus souvent reconstitué, à quelques kilomètres à peine des anciens postes frontières, des barrages de contrôle conduits par des patrouilles volantes, tandis que la police tchèque multiplie les contrôles dans l'ensemble du pays sous les noms évocateurs d'opération «Etranger» ou «Territoire». Surtout, les tendances amorcées depuis l'élargissement de l'UE en 2004 et conduisant à la cristallisation d'une nouvelle "géographie $d u$ crime 30 » et de la déviance dans le domaine de l'immigration semblent devoir se renforcer, après la disparition des postes frontières traditionnellement investis d'un pouvoir particulier dans la lutte contre l'irrégularité. L'extension, en République tchèque comme en Autriche, des possibilités de détention à des fins d'expulsion des demandeurs d'asile comme des migrants en situation irrégulière peut ainsi être envisagée comme une forme de réponse à la disparition $\mathrm{du}$ poste frontière comme lieu premier $\mathrm{du}$ «tri » des circulations. Alors que se dessine, entre 2004 et 2007, la perspective d'une frontière ouverte entre l'Autriche et la République tchèque, de nouveaux lieux d'enfermement apparaissent (dans la zone de transit des aéroports notamment), accompagnés d'une nouvelle rhétorique du contrôle de l'«étranger illégal » s'inscrivant directement dans le contexte de l'élargissement de l'espace Schengen et du déplacement consécutif des formes et des lieux du contrôle. C'est alors que prend tout son sens la mise en perspective, dans une ethnographie du contrôle migratoire, des formes de contrôle déployées dans les postes frontières avec celles mises en œuvre au sein des lieux de confinement des étrangers.

29. Cette représentation semble possiblement conditionnée par l'histoire mouvementée des zones frontalières de la Tchécoslovaquie et les politiques d'expulsion puis de repeuplement dont elles ont successivement fait l'objet. Sur ce point, voir Darley M.A., Frontière, asile et détention des étrangers. Le contrôle étatique de l'immigration et son contournement en Autriche et en République tchèque, op. cit.

30. Jobard F., "Que sait la police?», Vacarme, n7, 1999, disponible sur : http://www.vacarme.eu.org/article99.html, 11 octobre 2007. 